\title{
Basic astronomy as part of a general higher education in the developing world
}

\author{
John Baruch ${ }^{1}$, Dan Hedges ${ }^{1}$, James Machell ${ }^{1}$, \\ K. Norris ${ }^{2}$ and Chris Tallon ${ }^{1}$ \\ ${ }^{1}$ School of Informatics, University of Bradford, Bradford, U.K. BD7-1DP \\ email: john@telescope.org \\ ${ }^{2}$ Department of Education, Bradford College, Bradford, U.K. BD7-1AY
}

\begin{abstract}
This paper describes a new initiative in support of the aim of Commission 46 of the IAU to develop and improve astronomy education at all levels throughout the world. This paper discusses the ideal specification of a facility to support basic astronomy within education programmes which are delivered to students who have access to the Internet. The available robotic telescopes are discussed against this specification and it is argued that the Bradford Robotic Telescope, uniquely, can support many thousands of users in the area of basic astronomy education, and the resource is free.

Access to the Internet is growing in the developing world and this is true in education programmes. This paper discusses the serious problems of delivering to large numbers of students a web based astronomy education programme supported by a robotic telescope as part of a general education. It examines the problems of this form of teaching for teachers who have little experience of working with IT and little knowledge of basic astronomy and proposes how such teachers can be supported.

The current system (http://www.telescope.org/) delivers astronomy education in the language, culture and traditions of England. The paper discusses the need to extend this to other languages, cultures and traditions, although for trainee teachers and undergraduates, it is argued that the current system provides a unique and valuable resource.
\end{abstract}

Keywords. Telescopes, robotic telescope, astronomy education, e-learning, robot, developing countries

\section{Introduction}

When Tony Blair came to power in the UK in 1997 he said that his three priorities were "Education, Education and Education". It is education and not biology that separates us from our forefathers who chased mammoths across the frozen tundra of Europe in the last ice age. It is education and knowledge that empowers us to find effective routes to deal with the threats of AIDS and global warming and differentiates between those who are terrorized by voodoo, black magic or creationist ideas and those who have the framework to understand the evolution of diseases like bird flu, the aggregation of oil reservoirs or the awe inspiring vast universe that we inhabit.

Commission 46 of the IAU seeks to further the development and improvement of astronomical education at all levels throughout the world. One of the key problems for many students around the world and many teachers is the difficulty of teaching the basics of astronomy illustrating our position in the universe and the daily, monthly and yearly rhythms that our environment is subjected to. 


\section{Basic astronomy education for all}

The growth of cities with their high buildings, street lights and general light pollution, television, computer games and other distractions ensure that astronomy and the position of the Earth in the Universe barely enter into the consciousness of many young people. In many nations, the majority of the engineers and scientists were first brought to science by the accessibility of astronomy and the nightly spectacle of the stars. This is no longer the case and many nations particularly the developed nations are concerned about the future supply of engineers and scientists. In the 2006 House of Lords report on science teaching in schools in the UK they were most concerned about education in physics up to age 18 for the A level examination:

"The most problematic subject of all is physics, with the number of students opting to take the subject showing a precipitous fall since 1998. The numbers continue to fall and currently stand at less than 60 per cent of the total in the late 1980s. Moreover, the situation is far worse in some schools than these aggregated figures would suggest, as the Royal Society noted, "science take-up is strongly skewed at present, with half of all A-level entries in science coming from just 18 per cent of schools"

The fact that few people now sit under the stars at night admiring the glorious procession of the heavens means that the stars and basic astronomy are not part of life for the majority. To deliver an understanding of the basics of astronomy with its nightly spectacle requires education. An essential core of basic education is an understanding of the place of the Earth in the Universe and the processes which give us day and night, seasons, the phases of the moon, eclipses, comets and the annual cycle of the stars.

The United Nations Convention on the rights of the Child, accepted and signed by 192 of the states of the United Nations, which is more than any of the other Human Rights Conventions, states that all young people have the right to education and that:

"States Parties shall promote and encourage international cooperation in matters relating to education, in particular with a view to contributing to the elimination of ignorance and illiteracy throughout the world and facilitating access to scientific and technical knowledge"

Astronomy is a key part of that education.

\section{Educating teachers is the first step to Education for All}

For many developing countries this universal education even to primary level delivered free for all children as required by the Convention on the Rights of the Child is not immediately possible. They first have to educate people to the level necessary to be teachers and then to train them to be teachers. Few schools in the developing world have enough books at both primary and secondary levels and even fewer have access to the Internet. The situation in higher education and especially in teacher training appears much better. In 1998 UNESCO called for all teacher training institutions to include ICT skills within their courses, which included access to the Internet (UNESCO 1998). Tanzania is a typical developing country which in a report by UNESCO (2002) listed the problems of teacher training as:

- Low tutor competencies;

- Lack of a systematic tutors continuous professional development;

- Inadequate college management skills;

- Poor Information Communications Technology;

- Lack of competency based curriculum and curriculum materials. 
The International Institute for Communications and Development, a charity funded by the Governments of Holland, Britain and Switzerland, working with developing countries had connected up 44 Tanzanian Teacher Training Colleges to the Internet, installed computers and trained their technicians by the end of $2004 \uparrow$. The results of this are typically indicated in Reports in $2005 \ddagger$ and verbal reports in 2006 which indicated that at least the ICT aspects of teacher training had been greatly improved with Tanzanian students training to be teachers in the main Teacher Training institutions of Mzumbe and Dar Es Salaam having good access to Internet facilities. There still remain some problems associated with the cost of supplying adequate bandwidth to deliver good access. But it is probable that by this time (2007) all Tanzania's teacher training institutions are connected and effectively working on the Internet with their education programmes for pre-service teachers. It is also probable that with the lead being taken by the governments of the developed countries that the situation in Tanzania is reflected in most of the developing countries. Thus in 2007 most students training to be teachers will probably have some access to the internet during their course.

Another key problem for the training of teachers in the developing countries that was noted in the 2002 UNESCO report on Tanzania was the low tutor competencies. With the Internet available to many of the Teacher Training Institutions it is now possible to start to reap the benefits of ICT and Internet access that the UNESCO reports discussed in 1998 and 2004. The Bradford Robotic Telescope is part of that benefit.

\section{The Bradford Robotic Telescope is available for all}

The Bradford Robotic telescope http://www.telescope.org/ is an extensive web site for teaching and learning the basics of astronomy and the position of the Earth in the Universe. It is because it works at the basic level that it can handle an enormous number of users. The users work through the interactive e-learning programmes on the web site. These are really designed for under 16-year-old students, but there are extensive materials available to support teachers who are working at the limits of their expertise. The programmes with their range of interactive learning modules include the option for a learner to request observations to be taken by the Bradford Robotic Telescope to produce data that will support their learning.

These requests are all handled automatically by the telescope systems which schedule the observation and return the data to the user. The telescope is situated at one of the best observatory sites in the northern hemisphere. The Observatorio del Teide on the Island of Tenerife in the Canary Islands. The excellent weather in the Canary Islands means that the user will normally have their request returned within days.

The Bradford Robotic Telescope is already widely used. It has over 11000 registered users, but the system is extensive and registration is only required for those users who wish to have data returned to them. Registration provides an email address for the system to return the data. There are about 5000 individual visitors per day to the site, where multiple hits from the same IP address and access agent on a single day are called a single visit. The telescope has completed over 20000 user requests and returned data to the user. The telescope is widely used by developing countries with about 100 of the 140 countries of the 11000 registered users being regarded as developing countries. The number of users from these countries varies from over 170 in India, 86 in Brazil, 48 in Mexico, to around 30 countries with a single user which include Belize and Vanuatu. It

$\dagger$ See http://www.iicd.org/projects/articles/iicdprojects.2004-11-12.6571234687 $\ddagger$ See http://cs.joensuu.fi/cbe/ 
is not known how many of these are students in teacher training institutions but from the e-mail comments it is clear that students are one of the groups using the telescope system.

The objectives of IAU Commission 46 talk about astronomy education to all levels and it can only be assumed that this encompasses all levels of education and the complete range of our astronomical understanding. The UN Convention on the rights of the Child is more specific with a view to

"the elimination of ignorance and illiteracy throughout the world and facilitating access to scientific and technical knowledge."

\section{A resource to deliver basic astronomy education}

For a robotic telescope system to contribute to these objectives, especially the objectives contained in the Convention of the Rights of the Child it must be capable of supporting an education programme to deliver the basic framework of a modern understanding of our place in the Universe starting with the basic facts about the Sun and Earth, its Moon, the orbits of the planets and the local solar system. It must also be capable of delivering these to very large numbers of students. Initially, for the developing world, these students will be trainee teachers.

The basic knowledge associated with astronomy has been encapsulated in many ways, books like Astronomy for Beginners (Becan \& Becan, 2004) are one area, the Web, through the English National Curriculum for Science $2006 \dagger$ is another. A general search for basic astronomy will reveal hundreds of sites giving a wide range of knowledge. An understanding of astronomy starts with gravity and an understanding that the Earth is a sphere, followed by what causes day and night and the seasons, building upon these concepts opens the door to understanding so that mature students can continue themselves. It is these basic concepts that are so difficult to illustrate and which benefit greatly from the use of simulations on a computer screen and access to a telescope to confirm concepts. This is precisely the structure of the Bradford Robotic Telescope system.

The extensive web site which can be found at http://www.telescope.org/ includes a comprehensive e-learning site with 34 basic astronomy topics which include making requests for observations with the robotic telescope to support learning and understanding. The extensive web site includes animations, models and simulations. There is an extensive archive of images and a host of sensors and web cams surrounding the robot so that the user can see the day and night time sky. Every night a video is taken of the stars rotating around the pole star and another of the stars setting on Mount Teide. Users can see for themselves that although the Sun and Moon set at different places throughout the year the stars always set in precisely the same place.

\section{Detailed description}

The robot telescope itself is actually four telescopes each with CCD cameras, filter sets and automatic focussing. There is the constellation camera which takes large 40 degree panoramas of the sky. The cluster camera takes three degree images of the sky showing many smaller stellar clusters and nurseries along with full images of the Moon. The galaxy camera takes 20 arc minute images of deep sky objects and the planetary camera is currently used to ensure good tracking with the galaxy camera but its pixels cover 0.7 arc seconds of sky and it is intended to upgrade it into a planetary camera to

$\dagger$ Available at http://www.nc.uk.net/ 
look at brighter objects which include the planets and the International Space Station taking thousands of images and selecting those which show sub arc second resolution. The only thing that the system is, as yet, unable to do is to take images of the Moon and include the surrounding stars.

This assembly of optics and data processing is able to deliver the images to support study into all areas of basic astronomy, encouraging the users to adopt the scientific method to investigate ideas and check out the limits of their veracity.

\section{Compared to other robotic telescopes}

There are many robot telescopes operating in the world. Most of these are remotely controlled telescopes (e.g. http://slooh.com/ ) operating over the internet with a web cam for the user to see what they are doing. These are regarded as remote telescopes rather than robotic. Robotic telescopes like the Bradford Robotic telescope operate without interference from the user. The user submits a request, the request is scheduled by the telescope and the data returned to the user. Most of the remote telescopes charge for their use a given fee for a given time. Some of these remote telescopes are very large. The Faulkes and Liverpool telescopes are 2-metre aperture telescopes which can do serious research but their field is about 5 arc minutes and so they are unable to capture the full lunar image or the panorama of the constellations which is an important starting point for astronomy education. The Bradford Robotic telescope provides the background understanding for students to graduate to using these other systems.

Another great advantage of the Bradford Robotic telescope is that it can handle very large numbers of users this is only possible because unlike any of the other robotic telescopes it provides service observing, self scheduling the requests and by working at the most basic levels there are only about 25 objects requested by the great majority of users and these can be handled every night they are visible.

At the present time the telescope is free for international users, English users are expected to subscribe to the service and provide funds to cover the running costs on http://schools.telescope.org/. This is a service focussed precisely on the needs of teachers in England delivering the National Curriculum to students from the age of ten up to sixteen.

It is recognized that the general service is delivered in the language culture and traditions of England. This may be fine for some countries but it clearly is not ideal for most countries of the world. It is desirable to have the facility translated into other languages and linked into other cultures. It is understood that young people will learn better if the astronomy, whilst presenting a modern scientific views, links into the mythology and cultures of their nation. It is planned to seek funding to translate the site into the major European languages incorporating the cultures associated with those languages.

\section{Conclusion}

The Bradford Robotic telescope is an extensive e-learning web site supported by a robotic telescope. It offers a unique resource to support the general education of teachers in developing countries. It has all the facilities required for such general education and provides extensive support for both teachers and learners. 


\section{Acknowledgements}

We would like to acknowledge the support for this work from the Nuffield Foundation, PPARC, the Gatsby Trust and the Paul Instrument Fund of the Royal Society. We would also like to acknowledge the help given by Dr Faustin Kamuzora of Mzumbe University Tanzania.

\section{References}

Becan J., \& Becan S. 2004, Astronomy for Beginners, Writers and Readers Publishing, ISBN 0863169996

UNESCO 1988, UNESCO World Education Report, pp. 19-20

UNESCO 2002, In: Buyela Wepukhulu (ed.), Capacity building for Lead Teacher Training Institutions in Tanzania, UNESCO

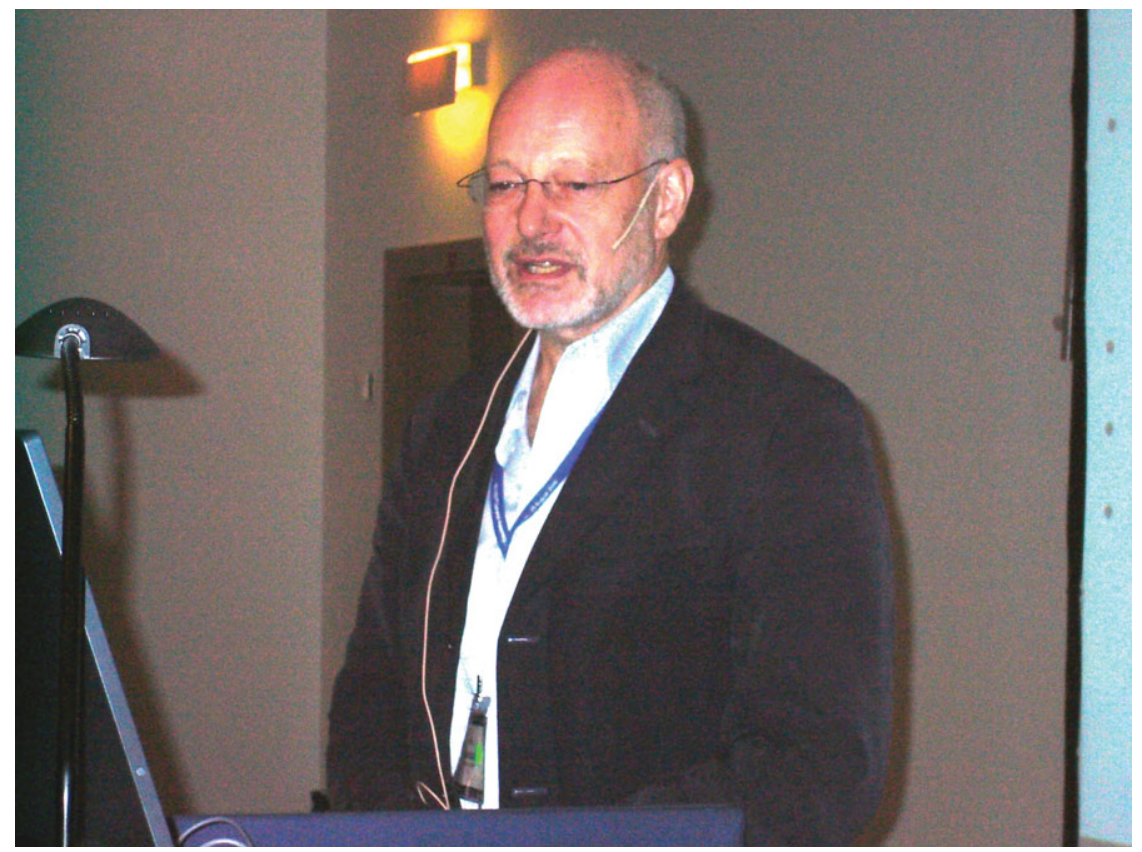

John Baruch 